\title{
Spontaneous Branching in a Polyp Oriented Model of Stony Coral Growth
}

\author{
Roeland Merks, Alfons Hoekstra, Jaap Kaandorp, and Peter Sloot \\ University of Amsterdam, Faculty of Science, \\ Section of Computational Science, \\ Kruislaan 403, 1098 SJ Amsterdam, The Netherlands \\ \{roel, alfons, jaapk, sloot\}@science.uva.nl, \\ WWW home page: http://www.science.uva.nl/research/scs/
}

\begin{abstract}
A three-dimensional model of diffusion limited coral growth is introduced. As opposed to previous models, in this model we take a "polyp oriented" approach. Here, coral morphogenesis is the result of the collective behaviour of the individual coral polyps. In the polyp oriented model, branching occurs spontaneously, as opposed to previous models in which an explicit rule was responsible for branching. We discuss the mechanism of branching in our model. Also, the effects of polyp spacing on the coral morphology are studied.
\end{abstract}

\section{Introduction}

Stony corals are colonial reef-building organisms, building an external calcium skeleton. The individual coral polyps reside in skeletal structures called corallites, and form a layer of living tissue surrounding the skeleton. Skeletal growth occurs as the polyps deposit new skeleton on top of the old skeleton (reviewed in [1]). In this process, the corallites move outwards, leaving behind a trail of coral skeleton. As the coral surface expands due to skeletal growth, the corallites move away from each other and divide, filling up the resulting space, a process called tissue growth (reviewed in [2]).

This seemingly simple growth process generates a wide range of colony morphologies; examples are spherical, thinly branched and plate-like forms. These morphologies are species specific, but also show high variability within one species. This intraspecific variability is caused by environmental parameters, such as light availability and the amount of water flow. In previous model studies, the effect of these environmental parameters on coral morphology has been assessed (light availability, $[3,4]$ and flow conditions, $[5,6]$ ). In the present study, we concentrate on morphologic differences between species; we keep the environmental parameters constant, and vary the properties of the individual polyps instead.

Our current work is based on the three-dimensional radiate accretive growth model $[6,7]$. In this model, coral growth is simulated by the accretion of discrete layers that are represented by a triangular mesh. The "coral" grows in a simulated fluid [6], in which nutrients are dispersed by advection and diffusion. The 
local thickness of the accreted layer depends on the flux of nutrients to the coral surface, which is a nutrient sink.

In this paper, we use a "polyp-based model", in which we model coral growth starting from the individual polyps. Coral growth is seen as the collective result of a process taking place in individual polyps. Each polyp takes up resources, deposits skeleton, buds off new polyps and dies. With this "polyp-based" approach, we have been able to make a number of simplifications relative to the model of Kaandorp et al. [6]. The most important of these is the omission of the socalled curvature rule. In the previous models this rule was necessary to generate branching growth forms. Here, we show that branching occurs without the curvature rule if we model the resource fluxes for each corallite invidually. In this way, a curvature effect comes out naturally. Relative to a plain surface, at a convex surface the corallites are fanned out and less of the resources near them are taken up by their competitors. At a concave surface polyps point towards each other and interfere strongly.

We concentrate exclusively on skeletal growth. In this paper we assume that the main rate limiting factor for skeletal growth is the availability of diffusing resources such as organic food or calcium ions. The rate of tissue growth is entirely dictated by the rate of skeletal growth, as it generates space in which new corallites are inserted.

In the remainder of this paper, we first present the "polyp oriented" model, and discuss some of the differences with the previous models. Then, we discuss the branching mechanism as it occurs in our model. After that, a parameter study is presented. We show a correlation between the spacing of the model polyps and the branch size in the final morphology.

\section{The Model}

We model the growth of the coral skeleton, which takes place at the living surface of the coral colony, using a triangular mesh. Diffusion of resources and other chemicals is modelled using the moment propagation method [8-10].

In short, a simulation step proceeds as follows. We start with the dispersion of resources. Since we use a grid based method, we first map the triangular mesh onto a three-dimensional grid using a voxelization method [11]. This grid-based representation of the mesh is then filled to generate a solid obstacle. Resources are dispersed from the top plane of the simulation box, and absorbed by the "polyps" . As soon as the influx of "resource" at the top plane balances the absorption of resources by the "polyps" (that is, if the resource field is stable), the resource flux $\phi_{i}$ at each "polyp" $p_{i}$ is measured. Now, we move back to the triangular mesh representation for an accretive growth step. On top of the previous layer, a new layer of skeleton is built, whose local thickness $l_{i}$ depends on the growth function $g\left(\phi_{i}\right)$. In the next sections these steps will be described in more detail.

\footnotetext{
${ }^{1}$ Throughout this text, the name of an entity $e$ will be put in quotes if it is used to denote "model of $e$ ".
} 


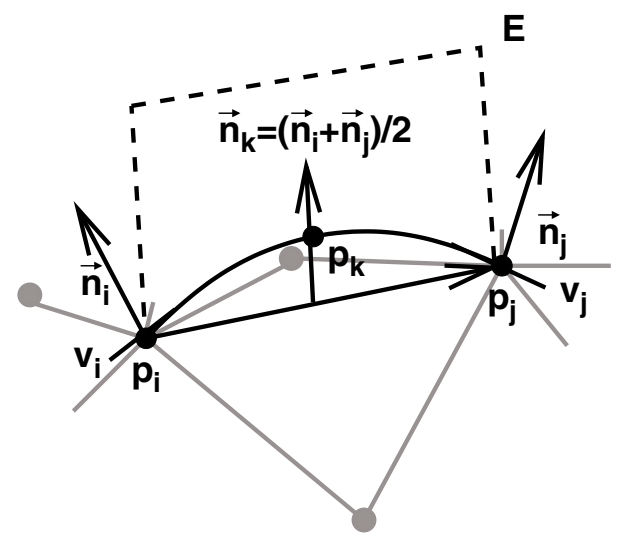

Fig. 1. Interpolation of the coral surface for "polyp" insertion. A third order polynomial $f$ is constructed, running tangential to the coral surface at $p_{i}$ and $p_{j}$. The new "polyp" $p_{k}$ is constructed at $p_{k}=\left(\frac{1}{2}, f\left(\frac{1}{2}\right), 0\right)$, relative to an orthonomal basis $B$ where $p_{i}=$ $(0,0,0), p_{j}=(1,0,0)$ and $z=0$ at plane $E$

\subsection{Accretive Growth}

As in the previous models of radiate accretive growth, the coral surface is described by a triangular mesh. In the present model, we model the individual coral polyps as the vertices $p_{i}$ of the triangular mesh. In contrast to the previous models, the triangles do not have a biological meaning. Each "polyp" $p_{i}$ consists of a coordinate $x_{i}, y_{i}, z_{i}$ in three-dimensional space and of one or more scalars $n_{i}$. In the present model, we use a single scalar $n_{i}$ which contains the resource influx $\phi_{i}$.

In each growth step, a longitudinal element $l_{i}$ is placed on top of each "polyp" $p_{i}$ along the mean normal vector of the surrounding triangles. The length of $l_{i}$ is determined by the growth function $g$. In the present work, this is a linear function of the resource flux $\phi_{i}$. The "polyp" $p_{i}$ is then moved to the end of the longitudinal element $l_{i}$, as if it pushes itself upwards by excreting calcium skeleton. In this process, the values of the scalars $n_{i}$ are retained.

After an accretive growth step, the length of the link $\boldsymbol{\Lambda}_{i j}$ between polyp $p_{i}$ and $p_{j}$ may have changed, depending on the curvature of the coral surface. A "polyp" is inserted or removed accordingly. If the distance $\left|\boldsymbol{\Lambda}_{i j}\right|$ between two "polyps" $p_{i}$ and $p_{j}$ drops below the fusion threshold $\theta_{\mathrm{FUSE}}$, the two polyps fuse. A new, fused "polyp" $p_{k}$ is placed at the middle of the interpolypal link, and the value of the scalars $n_{k}$ are set equal to the mean $n_{k}=\left(n_{i}+n_{j}\right) / 2$. Then, the "polyps" $p_{i}$ and $p_{j}$ are removed.

If the distance between two "polyps" $p_{i}$ and $p_{j}$ rises above the insertion threshold $\theta_{\text {INS }}$, a new polyp is inserted. The position of this polyp is determined using a third order interpolation of the coral surface (see fig. 1). As for "polyp" fusion, the values of the scalars is set to mean of the parent "polyps", $n_{k}=\left(n_{i}+\right.$ $\left.n_{j}\right) / 2$. Note that in case of "polyp" insertion, the parent "polyps" are retained in 

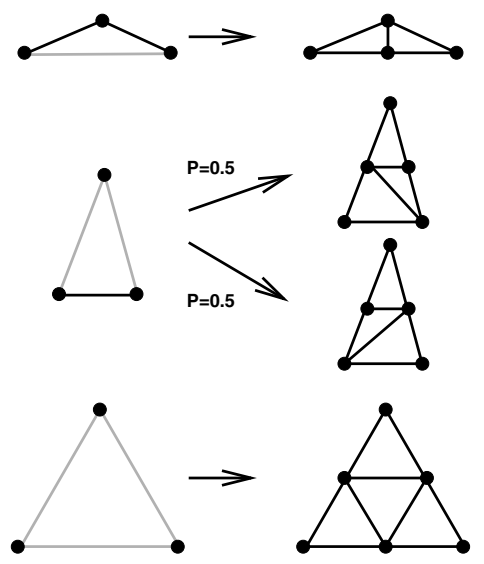

I.

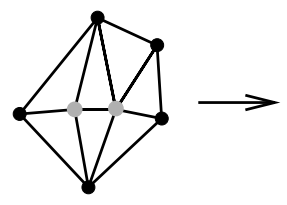

II.
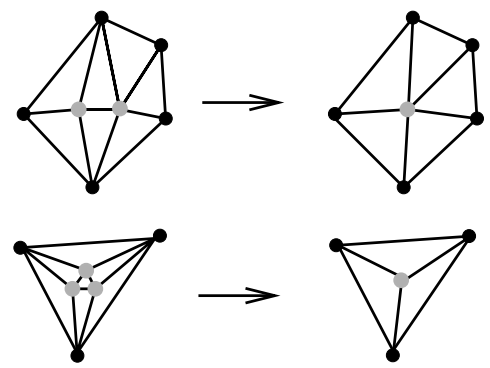

I.

II.

III.

Fig. 2. Triangle insertion (left) and fusion (right) rules. Gray links are divided. Gray nodes are fused

the simulation. The insertion and fusion threshold are set relative to the initial mean distance between "polyps", as $\theta_{\mathrm{FUSE}}=\frac{3}{4}\left\langle\left|\boldsymbol{\Lambda}_{i j}\right|\right\rangle$ and $\theta_{\mathrm{INS}}=\frac{3}{2}\left\langle\left|\boldsymbol{\Lambda}_{i j}\right|\right\rangle$, where $\left\langle\left|\boldsymbol{\Lambda}_{i j}\right|\right\rangle$ is the mean initial length of the interpolypal links.

As "polyps" are inserted and deleted from the simulation, new interpolypal connections are created and old ones are removed. In this way triangles are inserted and deleted from the mesh, according to the the triangle insertion and deletion rules. These rules are summarized in figure 2 .

\subsection{Resource Diffusion}

After the completion of an accretive growth step, the diffusion and the absorption of the limiting resource is simulated. We solve the diffusion equations on a cubic lattice. For this, the triangular mesh describing the surface of the coral colony is mapped onto the cubic lattice, for which we use the triangle voxelization method by Huang et al [11]. The resulting hollow "shell" is filled using a fast, heuristic three-dimensional seed fill algorithm.

The diffusion equation is solved using the moment propagation method [810]. This method is used in conjunction with the lattice Boltzmann method (reviewed in [12]), and has been shown to solve the advection-diffusion equation [9]. At each lattice node, a scalar quantity $P(x, t)$ is redistributed over the neighbouring nodes, according to the probability $f_{i}$ that a fluid particle moves with velocity $\boldsymbol{c}_{i}$ after collision,

$$
P(x, t+1)=\sum_{i} \frac{f_{i}\left(x-c_{i}\right) P\left(x-c_{i}, t\right)}{\rho\left(x-c_{i}\right)} .
$$

For a stationary fluid the velocity probabilities $f_{i}$ are identical for each lattice site, and depend only on the length of the lattice link. In the 19-velocity lattice we use, there are three types of links: one velocity 0 link, six links of velocity 
1 to the face-connected neighbours and twelve links of velocity $\sqrt{2}$ to the edgeconnected diagonal neighbours. For these links, $f_{i}=\frac{1}{3}, f_{i}=\frac{1}{18}$ and $f_{i}=\frac{1}{36}$, respectively.

\subsection{Coupling the Accretive Growth and Diffusion Models}

Simulation Set Up All our simulations are carried out on a grid of $200^{3}$ lattice units (l.u.). At the top plane the concentration of resources is fixed at 1.0, the ground plane is a resource sink. The other boundaries conditions are periodic. The initial condition is a hemisphere containing 81 "polyps" positioned at on the ground plane, where the center of the sphere is located at $(x, y, z)=(100,100,0)$. The radius of the initial hemisphere (and thus the spacing of the "polyps") varies over the presented simulations between 6.25 l.u. and 12.5 l.u.

Resource Absorption At each time step of the diffusion algorithm, the "polyps" absorb resource, thus acting as resource sinks. The position of each "polyp" in the simulation is mapped onto the cubic lattice. Since the "polyps" are part of the coral surface, the mapped positions have been marked as "solid" in the voxelisation algorithm. Therefore, the absorption takes place at a small distance $l_{\text {reach }}$ from the "polyp" position, normal to the coral surface. Throughout the simulations presented in this paper $l_{\text {reach }}=3$ l.u., thus ensuring that resources are absorbed at least from the first node marked as "fluid".

The diffusion algoritm is iterated until the influx of resources from the source plane balances the total outflux of resource into the "coral polyps" and the ground plane. We measure the resource influx $\phi_{i}$ for each "polyp" $p_{i}$ individually.

As the "coral" grows towards the top plane, the resoure fluxes $\phi_{i}$ increase. In order to fix the growth rate, the resource absorption is normalised against the maximum resource flux, $\phi_{i} \rightarrow \phi_{i} / \phi_{\max }$. The rationale behind this is that we fix the thickness of the boundary layer between the growing coral colony and the rest of the sea water, in which the nutrient concentration is assumed constant and constantly mixed.

\section{Results}

\subsection{Coral Branching}

In figure $3^{2}$ a possible outcome after 84 iterations of the diffusion limited accretive growth process is shown. The "coral" is grown upon an initial hemisphere of radius 6.25 l.u., giving a mean "interpolyp" distance of 1.9 l.u. Note that the growth form is strongly branched, although no special assumptions were made to get branching growth.

In previous models of accretive coral growth [6,13], an explicit curvature rule was necessary to enforce branching and branch splitting. This rule, simulating

\footnotetext{
${ }^{2}$ VRML files and MPEG movies of the objects shown in the figures have been made available at http://www.science.uva.nl/ ${ }^{\text {roel/ICCS2002 }}$
} 

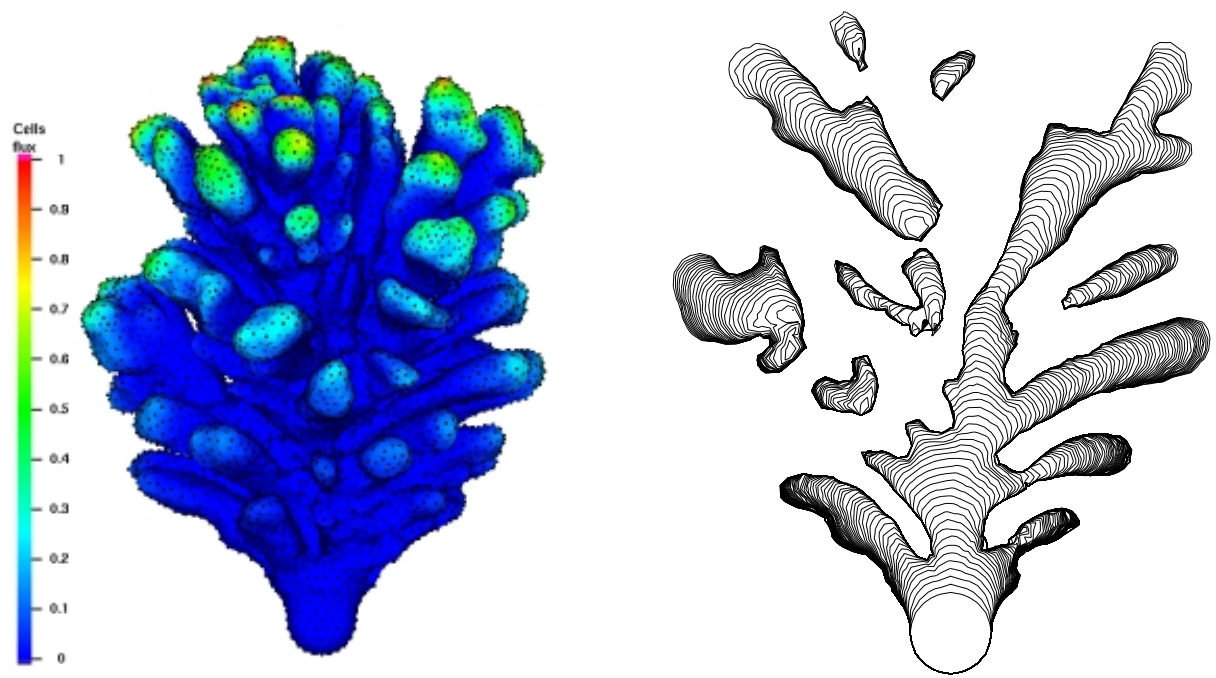

Fig. 3. Simulated branching coral after 84 growth steps. The size of the cubic grid is $200^{3}$. The coral contains 18006 "polyps", which are shown as black dots. The mean "interpolypal" distance is 1.9 l.u. Left panel: outside view, color scale: normalized resource flux. Right panel: cross section, in which the successive growth layers are visible

the amount of contact with the environment, increases the growth rate at places of high curvature. If the curvature drops below some positive threshold value, growth is completely suppressed. This procedure leads to a branching instability.

Here we show that branching occurs spontaneously if the resource fluxes are resolved for the "polyps" individually. In our model, there is no explicit curvature rule. Yet, branching is explained by a similar process. Absorption takes place at a distance $l_{\text {reach }}$ from the skeleton. This corresponds to real coral polyps, in which the mouth and tentacles are located at a small distance from the coral surface. At a convex location, the "polyps" fan out, thus getting better access to the resources than the "polyps" located at a flat or concave part of the "coral". At concave sites the resources are quickly depleted, because many "polyps" absorb resources from the same location and the resource replenishment is poor. Since the local deposition rate depends linearly on the resource flux, the local skeletal growth rate correlates positively with the local curvature which promotes branching. The precise correspondence between the curvature of the "coral" surface, the flux of resources into the "polyps" and the skeletal growth rate is the subject of ongoing research.

\subsection{Branch Size Depends on "Polyp" Spacing}

In section 3.1 we have shown that a curvature effect, caused by the "polyps" fanning out at convex sites, is responsible for the branching growth forms we 

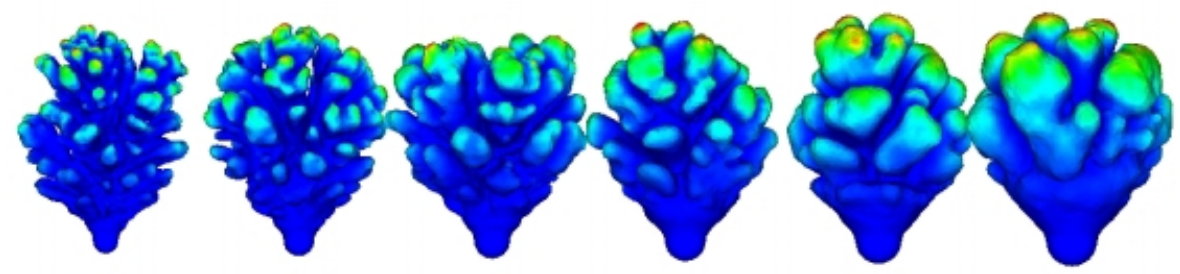

Fig. 4. Range of coral morphologies with increasing mean "intercorallite" distance. 85 growth steps. Initial sphere has 181 "polyps". Far left: "intercorallite" distance is 1.8 l.u. Far right: mean "intercorallite" distance is 3.7 l.u.

observe in our simulations. The more the absorbing ends of the "polyps" fan out, the higher the per polyp resource influx. One would therefore expect, that the mean "corallite" spacing modulates the curvature effect, which in turn affects the growth form. We have tested this hypothesis by starting with initial hemispheres of increasing radius, each of them having 181 "polyps". The initial spacing of the "polyps" dictates the mean "intercorallite" distance in the growth form, because the insertion and fusion threshold $\theta_{\text {INS }}$ and $\theta_{\text {FUSE }}$ are set relative to the intitial mean "intercorallite" distance.

In figure 4 a range of growth forms obtained after accretive growth steps is shown. At the far left, the mean "intercorallite" distance is 1.9 l.u., increasing to 3.7 l.u. at the far right. With increasingly wide "corallite" spacing, the branches become thicker and less numerous. Also, the branching split less quickly; the branch grows for a longer time before splitting up. The far right "coral" still branches like the "coral" at the left, though, which can be seen from figure 5 . In this experiment, we have continued the growth of the "coral" with a mean "intercorallite" distance of 3.7 l.u. until we obtained 150 growth layers.

\section{Conclusion and Discussion}

Branching: the Polyp Competition Hypothesis In the polyp oriented model, branching occurs as an emergent property. This finding contrasts previous models of branching coral growth, where an explicit curvature rule was introduced to enforce branching $[6,7,13]$.

Our model suggests a biological explanation for branching growth in stony corals. At convex sites, the polyps fan out, thus getting better access to the diffusing resources. At concave sites, the polyps point towards each other, thus interfering in the uptake of resources. In this way, a curvature effect comes out as a natural consequence of the competition between the polyps to take up resources from the water.

As the mean intercorallite distance increases (fig. 4), the coral branches less strongly. This observation fits well within the "polyp competition hypothesis" 

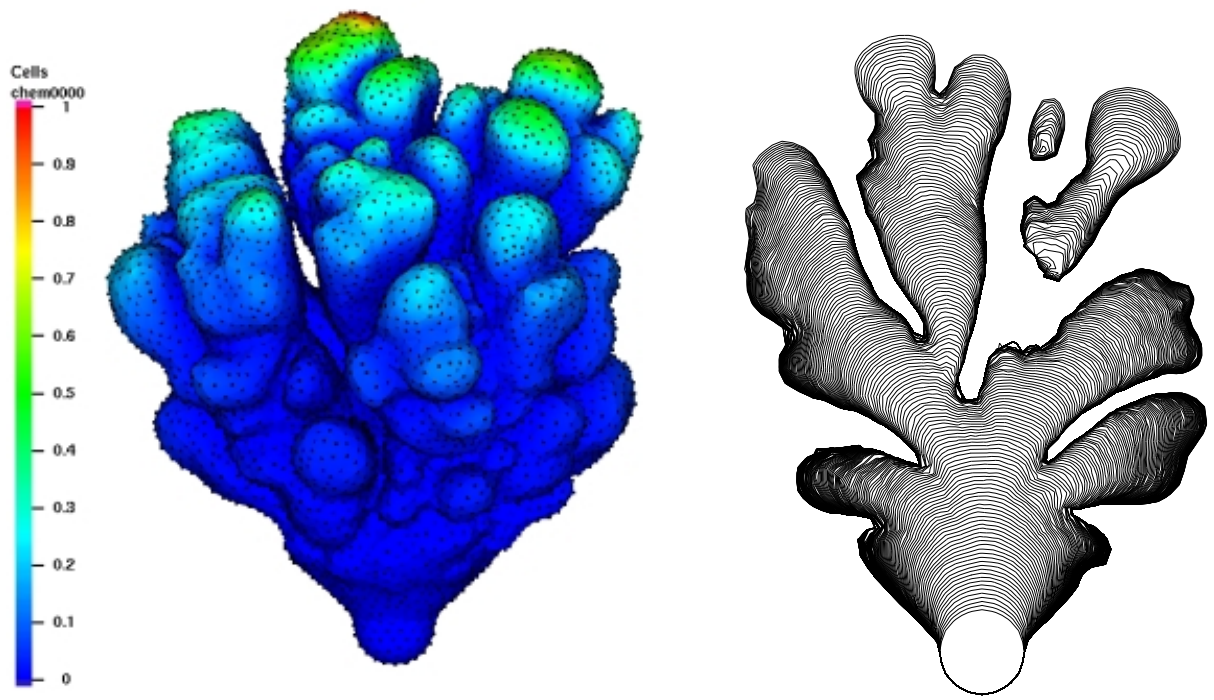

Fig. 5. Coral with initial "intercorallite" distance of 3.7 l.u. (fig. 4, far right) with 150 growth steps

that we proposed above. The competition for resources becomes less strong for larger mean "intercorallite" distances.

Polyp Oriented Modelling We have developed a polyp oriented approach to modelling coral growth. In this polyp oriented approach, branching occurs as a natural consequence of the competition for resources between the "polyps" at a curved surface. The polyp based approach will be useful in understanding how genetic information drives the morphogenesis of coral colonies. Genetics affects coral morphology indirectly, by setting the properties of the individual coral polyps. We can only come to an understanding of the genetic guidance of coral morphogenesis, through a thorough understanding of how the properties of individual polyps shape the coral colony. In the study of corallite spacing we have made a first step in this direction.

\section{References}

1. Buddemeier, R., Kinzie III, R.: Coral growth. Oceanography and Marine Biology: An Annual Review 14 (1976) 183-225

2. Harrison, P., Wallace, C.: Reproduction, dispersal and recruitment of scleractinian corals. In Dubinsky, Z., ed.: Coral Reefs. Ecosystems of the world. Volume 25. Elsevier Science Publishers B.V., Amsterdam (1990) 133-207

3. Graus, R.R., Macintyre, I.G.: Light control of growth form in colonial reef corals: computer simulation. Science (1976) 
4. Graus, R.R., Macintyre, I.G.: Variation in growth forms of the reef coral montastrea annularis (Ellis and Solander): A quantitative evaluation of growth response to light distribution using computer simulations. Smithsonian Contributions to the Marine Sciences (1982)

5. Kaandorp, J.A., Lowe, C.P., Frenkel, D., Sloot, P.M.A.: Effect of nutrient diffusion and flow on coral morphology. Phys. Rev. Lett. 77 (1996) 2328-2331

6. Kaandorp, J.A., Sloot, P.M.A.: Morphological models of radiate accretive growth and the influence of hydrodynamics. J. Theor. Biol. 209 (2001) 257-274

7. Kaandorp, J.A.: Analysis and synthesis of radiate accretive growth in 3 dimensions. J. Theor. Biol. 175 (1995) 39-55

8. Lowe, C.P., Frenkel, D.: The super long-time decay of velocity fluctuations in a two-dimensional fluid. Physica A 220 (1995) 251-260

9. Warren, P.B.: Electroviscous transport problems via lattice-Boltzmann. Int. J. Mod. Phys. C 8 (1997) 889-898

10. Merks, R.M.H., Hoekstra, A.G., Sloot, P.M.A.: The moment propagation method for advection-diffusion in the lattice Boltzmann method: validation and Péclet number limits. Submitted to Journal of Computational Physics (2001)

11. Huang, J., Yagel, R., Filippov, V., Kurzion, Y.: An accurate method for voxelizing polygon meshes. In: 1998 ACE/IEEE Symposium on Volume Visualization. (1998) $119-126$

12. Chen, S., Doolen, G.D.: Lattice Boltzmann method for fluid flows. Annu. Rev. Fluid Mech. 30 (1998) 329-364

13. Kaandorp, J.A., Sloot, P.M.A.: Parallel simulation of accretive growth and form in three dimensions. Biosystems. 44 (1997) 181-192 